\title{
Developing a machine learning model to identify protein- protein interaction hotspots to facilitate drug discovery
}

\author{
Rohit Nandakumar ${ }^{\text {Corresp., } 1}$, Valentin Dinu ${ }^{1}$ \\ ${ }^{1}$ Program of Biomedical Informatics, College of Health Solutions, Arizona State University, Tempe, Arizona, United States \\ Corresponding Author: Rohit Nandakumar \\ Email address: rnandaku@asu.edu
}

Throughout the history of drug discovery, an enzymatic-based approach for identifying new drug molecules has been primarily utilized. Recently, protein-protein interfaces that can be disrupted to identify small molecules that could be viable targets for certain diseases, such as cancer and the human immunodeficiency virus, have been identified. Existing studies computationally identify hotspots on these interfaces, with most models attaining accuracies of $\sim 70 \%$. Many studies do not effectively integrate information relating to amino acid chains and other structural information relating to the complex. Herein, 1) a machine learning model has been created and 2) its ability to integrate multiple features, such as those associated with amino-acid chains, has been evaluated to enhance the ability to predict protein-protein interface hotspots. Virtual drug screening analysis of a set of hotspots determined on the EphB2-ephrinB2 complex has also been performed. The predictive capabilities of this model offer an AUROC of 0.842 , sensitivity/recall of 0.833 , and specificity of 0.850 . Virtual screening of a set of hotspots identified by the machine learning model developed in this study has identified potential medications to treat diseases caused by the overexpression of the EphB2-ephrinB2 complex, including prostate, gastric, colorectal and melanoma cancers which are linked to EphB2 mutations. The efficacy of this model has been demonstrated through its successful ability to predict drug-disease associations previously identified in literature, including cimetidine, idarubicin, pralatrexate for these conditions. In addition, nadolol, a beta blocker, has also been identified in this study to bind to the EphB2-ephrinB2 complex, and the possibility of this drug treating multiple cancers is still relatively unexplored. 
6

7

8

9

10

11

12

13

14

15

1

2

3

$4{ }^{1}$ Program of Biomedical Informatics, College of Health Solutions, Arizona State University, Tempe,

\section{Facilitate Drug Discovery}

Rohit Nandakumar ${ }^{1}$; Valentin Dinu, $\mathrm{PhD}^{1}$
Arizona, United States of America

\section{Developing a Machine Learning Model to Identify Protein-Protein Interaction Hotspots to}

Corresponding Author:

Rohit Nandakumar

Address: Biomedical Informatics - ASU

Mayo Clinic, SC. Johnson Research Bldg

13212 E Shea Boulevard

Scottsdale AZ 85259

Email Address: $\underline{\text { nnandaku@asu.edu }}$ 


\section{ABSTRACT}

17 Throughout the history of drug discovery, an enzymatic-based approach for identifying new drug

18 molecules has been primarily utilized. Recently, protein-protein interfaces that can be disrupted to

19 identify small molecules that could be viable targets for certain diseases, such as cancer and the human

20 immunodeficiency virus, have been identified. Existing studies computationally identify hotspots on these

21 interfaces, with most models attaining accuracies of $\sim 70 \%$. Many studies do not effectively integrate

22 information relating to amino acid chains and other structural information relating to the complex. Herein,

23 1) a machine learning model has been created and 2) its ability to integrate multiple features, such as

24 those associated with amino-acid chains, has been evaluated to enhance the ability to predict protein-

25 protein interface hotspots. Virtual drug screening analysis of a set of hotspots determined on the EphB2-

ephrinB2 complex has also been performed. The predictive capabilities of this model offer an AUROC of

27

28

29

30

31

32

0.842 , sensitivity/recall of 0.833 , and specificity of 0.850 . Virtual screening of a set of hotspots identified by the machine learning model developed in this study has identified potential medications to treat diseases caused by the overexpression of the EphB2-ephrinB2 complex, including prostate, gastric, colorectal and melanoma cancers which are linked to EphB2 mutations. The efficacy of this model has been demonstrated through its successful ability to predict drug-disease associations previously identified in literature, including cimetidine, idarubicin, pralatrexate for these conditions. In addition, nadolol, a beta blocker, has also been identified in this study to bind to the EphB2-ephrinB2 complex, and the possibility of this drug treating multiple cancers is still relatively unexplored.

\section{$\underline{\text { INTRODUCTION }}$}

Drug discovery is the scientific process where new drugs and small molecules are developed and identified to treat certain conditions. Throughout most of the history of drug discovery, an enzymaticbased (lock and key) approach for identifying new drug molecules was utilized (Bakail \& Ochsenbein, 2016). As a result, many drugs targeting G-protein coupled receptors (GPCRs), which interact via this approach, constitute about $34 \%$ of the drugs in the market today (Hauser et al., 2017). 
43 EphA4-EphrinB2 complex, which is considered to be conformationally flexible (Ma \& Nussinov, 2014).

44 Protein-protein interfaces can be stabilized or disrupted to identify small molecules that could be viable

45 targets for certain diseases such as cancer and the human immunodeficiency virus (HIV). Identifying

46 residue hotspots on these protein-protein interfaces and repurposing existing drugs to target these new

47 hotspots can lead to novel drug targets, ultimately leading to new therapeutic treatments (Scott et al.,

48 2016). Although protein-based drug discovery (as opposed to enzymatic-based drug discovery) is a

49 relatively new and emerging field, recent studies have shown promising results in regard to its potential in

50 a wide range of fields from drug discovery to drug repositioning. For example, the SpotOn study has

51 produced remarkable results in regards to identifying hotspots that are viable for drug discovery, and

52 AnchorQuery, which identifies small molecule protein-interaction inhibitors. (Moreira et al., 2017; Koes,

53 Dömling \& Camacho, 2018)

54 In addition, PPI-based peptide drug discovery has been used to identify new therapeutic targets

55 by disrupting PPIs. Major advances in docking simulations and models in recent years have yielded to be

56 effective in more accurately identifying peptide-protein interactions. Although peptide-based PPI drug

57 discovery does have its challenges, such as limited bioavailability and solubility of peptides, this

58 emerging field highlights potentially exciting advances in computationally aided protein-protein

59 interaction based discovery techniques with the use of interfering peptides. (Lee et al., 2019)

60 Currently, only $10-14 \%$ of the human proteome is considered to be "druggable", and most targets

61 with published leads are in the rhodopsin-like GPCR family, with a smaller number in cation channels

62 and protein kinases (Hopkins \& Groom, 2002; López-Cortés et al., 2019). Druggability is the ability for a

63 drug to bind to a specific target. As protein-based drug discovery is a relatively new field compared to

64 traditional drug discovery, more research is needed to identify new hotspots on protein-protein interfaces.

65 Existing studies do computationally identify hotspots on these interfaces, but most of the models

66 developed only attain accuracies of around 70\% (Kim, Chivian \& Baker, 2004; Tuncbag, Keskin \&

67 Gursoy, 2010). Moreover, many studies do not effectively integrate information relating to amino acid

PeerJ reviewing PDF | (2020:05:49083:1:2:NEW 23 Sep 2020) 
68 chains and other structural information relating to the complex/interface, and/or have completely different 69 approaches to predict the likelihood of hotspots on a particular interface.

70 For example, molecular dynamics (MD) simulations have been used to elucidate the mechanisms 71 of protein interactions and their viability for drug discovery. This strategy has mixed results however 72 although the approach of molecular dynamics simulations have relatively high predictive power, these 73 simulations are computationally expensive (Cukuroglu et al., 2014). In contrast, knowledge-based 74 machine learning techniques have the advantage of providing accurate results based on the 75 properties/features of a specific interaction. Machine learning and other statistical approaches allow for a 76 high predictive power of hotspot detection, while being computationally efficient, provided that the 77 features inputted into the model are relevant.

This leads to the proposed research question, "Can the development of a machine learning model lead to the discovery of new druggable targets and new drug-disease associations?" The hypothesis was that the integration of different protein-protein interaction features will lead to promising new hotspots. In addition, new drug-disease associations could potentially be identified from these hotspots to treat deadly diseases such as cancer.

84 multiple features, including structural information, such as that associated with amino-acid chains, to enhance the ability to predict protein-protein interface hotspots was evaluated. In addition, virtual drug screening of a set of hotspots identified by the machine learning model developed herein was performed

87 in order to identify potentially new drug-disease associations. Phase 1 consisted of developing the machine learning model to identify potential protein-protein interface hotspots that could be viable as a drug target, using the cancer-associated EphB2-ephrinB2 protein complex (PDB code: 1KGY) for illustration. Phase 2 of this project aimed to identify small molecules that could act as inhibitors or

91 disruptors to the hotspots identified for further analysis in Phase 1.

92 The machine learning model developed in Phase 1 achieved an area under receiver operating characteristic (AUROC) of .842 on the testing set, and identified residues 1122-1126 on this complex as 
94 potential hotspot residues. This information was then used to generate a pharmacophore in Phase 2 which

95 identified nine drug candidates to disrupt the EphB2-ephrinB2 complex. Out of these candidates, further

96 literature review identified four drug candidates that could treat diseases that are overexpressed by this

97 complex: cimetidine, idarubicin, pralatrexate, and nadolol. Although nadolol has been relatively

98 unexplored in its potential of treating certain cancers, a drug with a similar chemical makeup, propranolol,

99 has been identified to treat multiple cancers including colon cancer, which is linked to the overexpression

100 of the EphB2-ephrinB2 complex, (Pantziarka et al., 2016) (Işeri et al., 2014), and thus highlights

101 significant repositioning opportunities for nadolol.

102

103 METHODS

104 Dataset Collection and Feature Aggregation

105 As a starting point, the dataset and codebase from the SpotOn study (Moreira et al., 2017) were acquired.

106 This study was selected as the starting point for its high effectiveness in identifying potential hotspots that

107 could aid in drug discovery. The SpotOn database already has information regarding amino acid

108 composition, solvent-accessible surface area (SASA) information, position-specific scoring matrices

109 (PSSMs), the number of amino acids at 2.5 and 4.0 Angstrom, the number of nearby hydrophobic

110 residues, the total change in solvent accessible surface area, the number of interfacial residues, pseudo-

111 amino acid composition, and scales-based descriptors of 2D and 3D descriptors from the protr R package

112 (see below) for a total of 881 features.

113 In order to add more information to this dataset to better aid model prediction, the protr R package (Xiao

114 et al., 2015) was used to add more features related to amino acid composition, dipeptide composition, etc.,

115 to the already pre-existing data. Additionally, data related to pair potential, complex/monomer accessible

116 surface area, residue information, amino acid information, etc. were extracted from the HotPoint database

117 (Tuncbag, Keskin \& Gursoy, 2010) and then added to the pre-existing dataset. This data was added to

118 add more information regarding the entire protein complex, as evidenced by most of protr's features, and 
119 to add residue specific features such as pair potential that could improve predictive power. The addition

120 of new features in the protr R package and the HotPoint database led to a total of 2323 features.

121 Upon further investigation of the SpotOn dataset, we found that chains I of proteins with PDB code

122 2FTL, 3SG8, and 1CH0 do not exist as specified in the Protein Data Bank. In the SpotOn study, these

123 chains are specified, and features were derived for these chains; however, in this study, as additional

124 features are added and these chains could not be identified, these chains have been removed from our

125 dataset. This leads to a total of 520 protein residues, lower than SpotOn's 534 protein residues. 398

126 residues are labelled as non-hotspots, and 122 residues are labelled as hotspots.

127 In order to derive features on our prediction dataset with the EphB2-ephrinB2 complex (PDB code:

128 1KGY), we first downloaded the structure from the Protein Data Bank, and ran this structure through the

129 SpotOn's codebase/pipeline to collect features specific to the SpotOn study. Then, we sequentially added

130 additional features unique to this study, such as from the protr's R package and features from the

131 HotPoint database. Missing values are assigned the average value of all non-missing values in a feature.

Preprocessing and Feature Engineering

134 Similar to the SpotOn study, both the training and testing sets were normalized, and the testing set was 135 normalized using mean and standard deviation of the training set. In addition, before the model was run, 136 data balance had to be accounted for, and oversampling was performed in order to retain the properties of 137 the majority class without sacrificing the information available in this class (More, 2016). SMOTE, or 138 synthetic minority oversampling technique, was performed with $\mathrm{k}=5$ nearest neighbors. (Chawla et al., 139 2002) To account for multicollinearity, principal component analysis was also performed. This leads to

140 four different combinations: a pipeline without any changes to the training data, a pipeline with only

141 SMOTE applied, a pipeline with only PCA applied, and a pipeline with both SMOTE and PCA applied.

143 Before the model was trained, the dataset was first subjected to feature engineering. Three existing

144 features that were selected for further exploration are the number of intermolecular contacts within 4.0 
145 Angstroms (\#Dist-4.0), the number of hydrophobic contacts (\#Hydrophobic), and the pair potential of a

146 specific residue (Pair Potential). We hypothesized that an increase of hydrophobic contacts would cause a

147 decrease in hydrophobic pair potential due to the attractive interaction because of the hydrophobic effect

148 (Israelachvili \& Pashley, 1982). As a result, we multiplied both variables and multiplied by -1 to amplify

149 the effects of this association and accounting for the inverse correlation. In addition, we hypothesized

150 that the number of intermolecular contacts will increase the pair potential as this may lead to many body

151 potentials, which are mostly repulsive at short distances (Byggmästar, Granberg \& Nordlund, 2018). To

152 model this association, \#Dist-4.0 and \#Hydrophobic are multiplied to amplify the effects as well. These

153 two new engineered variables were named \#Dist-4.0 * Pair Potential and -\#Hydrophobic * Pair

154 Potential. This lead to a total of 2323 features on the training and testing datasets, as well as our dataset

155 containing residue information on the crystal structure of the EphB2-ephrinB2 complex (PDB code:

$1561 \mathrm{KGY})$.

157

158

Machine Learning Model Selection

159 Five different machine learning models were selected in order to evaluate and develop a model: Logistic

160 regression (LR), XGBoost (XGB), a balanced random forest classifier (RF), K Nearest Neighbors (KNN),

161

162

163

164

165

166

167

168

169

170 multilayer perceptron neural network (MLP), and a Gaussian Naïve Bayes (GNB). This data was then split into a training:testing set ratio of 80:20. 10-fold cross validation was performed 10 times on the training set to prevent overfitting. GridSearch was performed in order to identify the best combination of hyperparameters/parameters that could yield the best results. The following hyperparameters/parameters were tested: LR, with C equal to .01, .1, 1, 10, 50, 100, 500, 1000, and 5000; RF with balanced class weight, with the number of estimators equal to 50,100,150,250,350,500, and maximum depth of 5, 7 , 9,11 XGB, with a learning rate of $.001, .01, .1$, the number of estimators as $50,100,150,200$, and maximum depth of 4, 5, 6; KNN, with n neighbors of $1,3,5,10,15,20$; a multilayer perceptron model of hidden_layer_sizes $(10,10,10),(50,1),(10,10),(10,1),(5,5),(5,5,5)$ and alpha of $0.0001,0.0002$, $0.0005,0.001$; and GNB with variance smoothing of $1 \mathrm{e}-8,1 \mathrm{e}-7,1 \mathrm{e}-6,1 \mathrm{e}-5,1 \mathrm{e}-4,1 \mathrm{e}-3$, and $1 \mathrm{e}-2$. The

Peer] reviewing PDF | (2020:05:49083:1:2:NEW 23 Sep 2020) 
171 metric used to identify the best model from these sets of parameters on the validation set is AUROC (area

172 under receiver operating characteristic), and this was chosen to compare our models more accurately with

173 the AUROC and receiver-operating characteristic curves provided in SpotOn. Four different run

174 conditions on the four different pipelines was also run and the results are compared. The model(s) with

175 the best run conditions on the highest scoring pre-processing dataset will be used to build an ensemble

176 model, similar to the SpotOn study. If the ensemble model has a higher predictive capability than any

177 individual model, the ensemble model will then be used to predict hotspots on the EphB2-ephrinB2

178 complex, as this complex has been overexpressed in many cancer cells, most notably in prostate, gastric,

179 colorectal and melanoma cancers. (Pasquale, 2010) PyMol was utilized to visualize the hotspots predicted

180 on the EphB2-ephrinB2 complex.

181

182 Small Molecule Selection

183 A cluster of hotspots was identified and LigandScout (Wolber \& Langer, 2005) was used to create an apo184 site pharmacophore. Virtual screening was then performed on this pharmacophore to identify possible 185 new drug indications. To perform the drug screening, an approved Drugbank (Wishart et al., 2008) 186 database that has a library of all molecules that have molecular weight from 150 to 500 daltons was used. 187 These small molecules were then ranked by the LigandScout software to identify molecules that most 188 strongly conform to the pharmacophore based on the chemical and structural properties of that molecule.

189 The drug-disease associations were then verified with scientific literature to assess the validity and 190 efficacy of the model, and then we identified new drug-disease associations that have not been previously 191 identified by cross-referencing existing scientific literature.

192

193 Metric Calculation

194 In context, sensitivity is the ability for the model to identify the hotspots and the specificity/recall is the 195 ability for the model to identify the non-hotspots, and both of these statistics are defined as: 
196

197

198

199

200

201

202

203

204

205

206

207

208

209

210

211 RESULTS

212 Phase 1

213 Table 1: Average test metrics of algorithms tested on pre-processing pipelines

214

215 The average test metrics of each of the six algorithms tested on the 4 different pre-processing pipelines

216 are shown in Table 1. As the preprocessing pipeline where SMOTE and PCA are applied has the highest

217 AUROC, the top algorithms from this pipeline are used to create an ensemble model. 
219 Table 2: Best Individual Algorithms in SMOTE and PCA pipeline

220

221 In Table 2 are the best individual algorithms tested in the SMOTE and PCA pipeline. The best set of 222 hyperparameters were selected using GridSearch as follows: the logistic regression with $\mathrm{C}=1000$, the 223 random forest classifier with maximum depth of 5 trees and the total number of estimators at 50 trees, an 224 XGBoost classifier with learning rate 0.1, maximum depth of 5, and 50 estimators, K-nearest neighbors 225 with 5 neighbors, a multi-layer perceptron classifier with alpha as .0002 and three layers of 10 neurons 226 each, and a Gaussian Naïve Bayes of variable smoothing of 1e-5. The best performing algorithm in this 227 pipeline is the logistic regression, as it has the highest AUROC.

228

229

Table 3: Comparison of our study vs SpotOn

230

231

*This data was adapted from the SpotOn study

232

The results of the Logistic Regression from the SMOTE and PCA pipeline were compared with SpotOn's highest performing algorithm from the upsampling pre-processing procedure. In order to more aptly analyze the predictive capabilities of our top performing algorithm, we adjusted the threshold to achieve a $>=.88$ specificity, as that is the specificity of the highest performing individual algorithm in SpotOn, and comparing other metrics based on this threshold change. This reduced the AUROC from 0.842 , as identified in Table 2, to 0.840 , as identified in Table 3, but was still greater than any individual model in demonstrated in Table 3.

241 Table 4: Different ensemble classifiers (stacking and voting) were tested 
243 The top ranking algorithms in the SMOTE and PCA pipeline are used to develop an ensemble classifier to

244 achieve better performance compared to any single algorithm. Different ensemble algorithms are tested:

245 stacking, where a meta-classifier is used to combine the predictive power multiple base classifiers, and

246 voting, a simple ensemble method where each of the six algorithms tested votes on a specific data point,

247 and a simple majority vote is used to predict the classification of that data point. In this case, the meta-

248 classifier used during stacking is a Logistic Regression classifier where $\mathrm{C}=5$. Each individual model is

249 used as a base model separately with the meta-classifier, and all models are combined with the meta-

250 classifier. All ensemble models are run on the SMOTE and PCA pipeline. In the voting ensemble, hard

251 voting was implemented, and all six algorithms are subjected to majority voting. Here, the best

252 performing classifier was the stacking classifier where all models are combined with the meta-classifier.

253 However, the AUROC of this ensemble method is still lower than that of the top individual model, the

254 logistic regression in the SMOTE and PCA pipeline.

255

256

Table 5: Comparison of our study to other studies

257

258

* Columns 2 through 7 are adapted from the SpotOn study to perform the side-by-side comparison among the algorithms

259 A comparison of the accuracy and performance of the model developed herein, shown in bold, compared 260 with SpotOn. In our model, the logistic regression was our top performing algorithm, and was thus used 261 to develop to predict hotspots with high accuracies. The SpotOn study (Moreira et al., 2017) was used in 262 order to identify the testing accuracies of the SpotOn study and those of the other studies as well. The 263 other studies that are compared to are SpotOn, SBHD213, Robetta23, KFC2-A24, KFC2-B, and 264 CPORT25. (Kim, Chivian \& Baker, 2004; Martins et al., 2014) (de Vries \& Bonvin, 2011; Zhu \& 265 Mitchell, 2011)

266

267

Phase 2

Table 6: Pharmacophore fit rankings from drug screening: Details for top ranked drugs 
269 The top logistic regression algorithm from the SMOTE and PCA pipeline was utilized to predict the 270 hotspots from the EphB2-ephrinB2 complex. This resulted in consecutive residues 1122-1126 predicted 271 as viable hotspots. Drug screening performed on these hotspots results in nine potential small molecules 272 that could bind to this hotspot. The pharmacophore fit scores are provided from our drug screening 273 analysis, where the highest ranked drug also has the highest pharmacophore fit score. Extensive literature 274 review was conducted to identify which drugs could aid in treating conditions associated with the EphB2275 ephrinB2 complex.

277 Phase 1

278 Figure 1: Feature importances of the top logistic regression classifier

279

280

Sensitivity analysis of the logistic regression from the SMOTE-only pipeline were performed. Features near the top of the graph 281 have higher feature importances.

282 As the best performing classifier, the logistic regression from the SMOTE and PCA pipeline is subjected 283 to PCA, it is difficult to analyze exactly which features are the most valuable. As a result, in order to 284 identify the most relevant features, sensitivity analysis using Python library Pytolemaic 285 (https://pypi.org/project/pytolemaic/) was performed on the best performing logistic regression classifier 286 from the SMOTE-only pipeline to understand the significance of adding new features to the existing 287 dataset as provided by the SpotOn study. Three out of the top ten features (Relative Complex ASA, 288 Complex ASA, and the engineered features Dist-4.0*Pair Potential) as identified by Figure 1 were added in this study exclusively, and highlights the improvement in predictive capabilities of the addition of these 290 features.

291 
295 PyMol (Delano WL, 2002) was used to derive and highlight residues 1122-1126 on chain E of the 296 EphB2-ephrinB2 complex. Predicted druggable hotspot residues are shown as more visible surface 297 markers (in green), and the other residues are shown in pink or light red. Residues 1122-1126 were 298 selected for drug screening as consecutive residues may be used as initial fragments in drug screening. 299 (Modell, Blosser \& Arora, 2016)

300

301

302 Phase 2

303 Figure 3: Apo-Site Grid for residues 1122-1126

304

305

Apo site pharmacophore of residues 1122-1126. The gray parts of the grid indicate the levels of buriedness and surface area. An apo-site grid was developed and implemented on hotspot residues 1122-1126 as identified via the

307 308 309 310 machine learning model on the EphB2-ephrinB2 complex, where a visual representation of the hotspots on the complex are shown in Figure 2. This grid was developed by first calculating the pockets of hotspot residues 1122-1126 on LigandScout (Wolber \& Langer, 2005).

\section{Figure 4: Pharmacophore model of residues 1122-1126}

This figure shows the 26-feature pharmacophore developed using an apo-site grid derived using hotspot residues 1122, 1123, 1124, 1125, and 1126 identified in Figure 3 via the machine learning model. A pharmacophore identifies the key parts of the molecular features that define the function and shape of a specific ligand, and includes features such as H-bond acceptors and donors, hydrophobic and aromatic rings, etc. This pharmacophore is then used to identify drugs that fit its features. The scoring of this screening procedure follows a pharmacophore-fit scoring function as provided in LigandScout. A maximum number of two features are omitted from this multi-feature pharmacophore to identify small molecule hits, and the best matching conformation is selected.

Peer] reviewing PDF | (2020:05:49083:1:2:NEW 23 Sep 2020) 
323 Figure 5: Structure and relative structure of cimetidine in relation to the developed pharmacophore

324 Cimetidine, currently an acid reflux medication, was identified via virtual screening to potentially bind to 325 the EphB2-ephrinB2 complex associated with cancer cells. The right image is cimetidine in relation to 326 the 26-feature pharmacophore developed as shown in Figure 4. A pharmacophore-fit score of 43.86 was 327 achieved during drug screening. Further literature review identified cimetidine as a potential 328 repositioning target for many different types of cancers, including melanoma, gastric, and colorectal 329 cancers. (Pantziarka et al., 2014)

330

331

Figure 6: Structure and relative structure of idarubicin in relation to the developed pharmacophore 333 Idarubicin, a chemotherapy medication that's currently used to treat breast cancer, was identified via virtual screening to potentially bind to the EphB2-ephrinB2 complex, where the expression of the complex is associated with cancer cells. The pharmacophore fit score of this small molecule is 45.46 . This drug was also found to treat cancers linked to the EphB2-ephrinB2 complex such as melanoma and leukemia. (Martoni et al., 1986) (Jabbour et al., 2017) The right image is idarubicin in relation to the pharmacophore developed as shown in Figure 4.

341 Figure 7: Structure and relative structure of pralatrexate in relation to the developed

\section{2 pharmacophore}

344 Pralatrexate, a T-cell lymphoma medication, was identified via virtual screening to potentially bind to the

345 EphB2-ephrinB2 complex, where the expression of the complex is associated with cancer cells. This

346 small molecule has a pharmacophore fit score of 47.41, and literature review suggests that this drug could 
347 potentially treat breast cancer and prostate cancer. (Yu, Zhao \& Gao, 2018) (Serova et al., 2011) Recent

348 research has shown that pralatrexate can treat esophagogastric cancer, which is associated with the

349 various gastrointestinal cancers of the EphB2-ephrinB2 complex. (Malhotra et al., 2020) The right image

350 is pralatrexate in relation to the pharmacophore developed as shown in Figure 4.

351

352 Figure 8: Structure and relative structure of nadolol in relation to the developed pharmacophore

353

354

355

356

357

358

359

360

361

362

363

364

365

366

367

368

369

370

371

372
Nadolol, a beta blocker, was identified via virtual screening to potentially bind to the EphB2-ephrinB2 complex, where the expression of the complex is associated with cancer cells. This small molecule has a pharmacophore fit score of 45.97 , and literature review suggests that beta blockers could potentially treat a variety of cancers, including breast cancer and pancreatic cancer. (Ishida et al., 2016) A close relative of this drug, propranolol, can induce apoptosis in liver cancer cells. (Wang et al., 2018) This research suggests nadolol's potential role in mitigating the effects of other cancers as well. The right image is nadolol in relation to the pharmacophore developed as shown in Figure 4.

Virtual drug screening identified nine drugs (pralatrexate, chlortetracycline, nadolol, imipenem, idarubicin, valganciclovir, conivaptan, cimetidine, and barnidipine) that bind to the pharmacophore shown in Figure 4. Further analysis via literature review identified four drug candidates to potentially treat various types of cancers: cimetidine, idarubicin, pralatrexate, and nadolol. Figure 5 shows the possibility for cimetidine, an antacid, to bind with the EphB2-ephrinB2 complex, and scientific literature identified the possibility for this drug to potentially treat melanoma, gastric, and colorectal cancers (Pantziarka et al., 2014). Figure 6 identifies the possibility for idarubicin, a chemotherapy drug used to treat leukemia, to bind with the EphB2-ephrinB2 complex, and literature review identified the possibility for this drug to potentially treat melanoma and leukemia (Martoni et al., 1986) (Jabbour et al., 2017). Figure 7 demonstrates the possibility for pralatrexate, a T-cell lymphoma medication to bind to the EphB2-ephrinB2 complex. 


\section{DISCUSSION}

375 In this paper, we presented our development of a machine learning approach for identifying druggable

376 hotspots at protein-protein interfaces. Our algorithm builds on previously existing methods, most notably

377 the SpotOn study. Our approach combines molecular features that have not previously been combined,

378 such as the molecular descriptors used in the SpotOn and HotPoint studies, and additional information

379 related to amino acid composition as provided by the protr module. It applies various machine learning

380 techniques, such as 10-fold cross-validation, feature engineering, and ensembling techniques, including

381 voting and stacking. A logistic regression with $\mathrm{C}=1000$ was used in order to achieve an AUROC of

3820.842 , sensitivity/recall of 0.833 , and specificity of 0.850 .

In order to find the most optimal pipeline, all four pipelines were run, and the pipeline that used

384

385

386

387

388

389

390

391

392

393

394

395

396

397

398

SMOTE and PCA during the pre-processing step was chosen the most optimal pipeline due to its high average AUROC score. The average metrics of all classifiers in each of the pre-processing steps are recorded in Table 1. Furthermore, the results of each top performing classifier in the SMOTE and PCA pre-processing step are illustrated in Table 2. In Table 3, the top metrics of the highest performing algorithm in the most optimal pipeline, the SMOTE and PCA pre-processing pipeline, are compared with the top-performing model in the ScaledUp pre-processing dataset in SpotOn, the highest performing dataset in that study. SpotOn-specific metrics are provided by the study itself. The individual models of our study performed better than the individual models of SpotOn as highlighted in Table 3. After this step, ensemble methods such as stacking and voting were implemented to potentially achieve even better results than any single model. The results of performing this step are shown in Table 4.

Although our models outperform that of SpotOn's individual models on all metrics, the results of our approach are lower on three out of four metrics than the top performing ensemble model from the SpotOn study, as illustrated in Table 5. This may be due to one of many reasons. Even though there was an increase in the total number of features as compared to the SpotOn study, the slight decrease in the total number of samples could potentially negatively affect predictive performance. Another reason could 
399 be that the models tested are not diverse enough from each other to significantly boost performance via

400 ensembling. Two of the models in this study are tree-based methods (random forest and gradient

401 boosting). This seems to be the most plausible explanation for why our top individual model

402 outperformed SpotOn's top individual model on all metrics, but ensembling techniques did not improve

403 performance. A greater diversity of these models would probably have boosted performance during

404 stacking or voting, as a greater variety of base models have been shown to boost predictive performance.

405 (Whalen \& Pandey, 2013)

406 To illustrate our approach, we applied this model to analyze the EphB2-ephrinB2 complex, which

407 has been overexpressed and associated with multiple types of cancer, including prostate, gastric,

408 colorectal and melanoma cancers. (Pasquale, 2010) As the overexpression of the EphB2-ephrinB2

409 complex is associated with these cancers, further analysis for drug discovery could aid in identifying

410 possible new hotspots that potentially aid in drug discovery in the fight against cancer (Barquilla \&

411 Pasquale, 2015). In addition, the viability for the EphB2-ephrinB2 complex, and more specifically the

412 EphB2 receptor, for drug discovery has been examined, and it was determined that small molecules could

413 potentially disrupt and/or bind to the ephrin binding pocket. (Chrencik et al., 2007) (Noberini, Lamberto

414 \& Pasquale, 2012)

415 The effectiveness of introducing new and engineered features was demonstrated by the

416 sensitivity analysis on the logistic regression on the SMOTE-only pipeline (Figure 1), where three out of

417 the top ten features were added in this study exclusively. Our algorithm identified a set of residue

418 hotspots (Figure 2), which were then used to generate an apo-site grid and pharmacophore model (Figure

4193 and Figure 4). This model was used to identify drugs with similar characteristics that could be

420 potentially used to modulate the molecular functions of the EphB2-ephrinB2 complex. Table 6 outlines

421 the nine small molecules that passed the drug screening test. Extensive literature review was performed

422 on all nine drugs, and four small molecules were selected their potential efficacy regarding their ability to

423 treat conditions associated with the EphB2-ephrinB2 complex. The identified drugs included compounds

424 already used for cancer treatment, such as pralatrexate, a T-cell lymphoma medication, as well as non-

Peer] reviewing PDF | (2020:05:49083:1:2:NEW 23 Sep 2020) 
425 cancer medication; cimetidine, an antacid; and nadolol, a beta blocker that can treat cardiac conditions.

426 Literature review suggests that pralatrexate can potentially treat breast cancer and prostate cancer, and

427 recent literature highlights the possibility for this small molecule to treat other conditions such as cancers

428 of the gastric and esophageal systems. (Yu, Zhao \& Gao, 2018) (Serova et al., 2011) (Malhotra et al.,

429 2020) Figure 8 identifies nadolol, a beta blocker that can treat cardiac conditions, as a candidate to bind

430 to the EphB2-ephrinB2 complex. Literature review strongly supports that beta blockers can be

431 repositioned to treat other cancers, such as cancer, and has identified a close relative of nadolol,

432 propranolol, as a potential treatment against multiple cancers, including colon cancer. (Işeri et al., 2014)

433

\section{CONCLUSION}

435 The model developed herein in phase one compares favorably with those developed in prior studies and offers enhanced predictive ability for identifying new druggable hotspots, including possible druggable

437

438

439

440

441

442

443

444

445

446

447

448

449

450 hotspots for cancer-related protein interfaces. The predictive capabilities of the model developed herein are high, offering a high AUROC and overall predictive performance to date. Herein, a logistic regression with $\mathrm{C}=1000$ was utilized to successfully identify hotspots.

Phase two of this project aims to identify possible drugs for repositioning. Structural properties of the identified hotspot residues, such as H-bond acceptors and donors, were identified as feature sets to aid in drug development. The efficacy of the model developed herein has been demonstrated through its successful ability to predict drug-disease associations previously identified in literature, including cimetidine, idarubicin, and pralatrexate. Importantly, nadolol has been uniquely identified in this study to potentially treat conditions caused by the overexpression of the EphB2-ephrinB2 complex. This work aims to yield better predictions in terms of hotspot discovery by primarily increasing the sheer amount of data that is available regarding protein-protein interactions. As a consequence, this work has shown that the increases in predictive power as a result of this addition of data.

49 Possible avenues for future work include drug development using the pharmacophores identified in this study to treat these diseases. Hopefully, by identifying hotspot residues with unparalleled accuracy and 
451 identifying possible drug repositioning opportunities, traditional drug development based on these

452 residues and repositioned drugs could yield new and effective treatments for diseases such as cancer. In 453 addition, adding additional novel features and data for hotspot identification, especially those that directly

454 correlate with the extent of how energetically favorable residues are, could further improve model

455 performance. Another avenue for future work would be to streamline the workflow of both phases.

456 Phase one is automated with the help of the machine learning model. However, phase two requires

457 manual input of the hotspot residues as identified in phase one to identify potential drug candidates. A

458 more streamlined process would improve functionality and ease of use.

459

460

461

462

463

464

465

466

467

468

469

470

471

472

473

474 Cukuroglu E, Engin HB, Gursoy A, Keskin O. 2014. Hot spots in protein-protein interfaces: Towards 475 476

\section{REFERENCES}

Bakail M, Ochsenbein F. 2016. Targeting protein-protein interactions, a wide open field for drug design. Comptes Rendus Chimie 19:19-27. DOI: 10.1016/j.crci.2015.12.004.

Barquilla A, Pasquale EB. 2015. Eph Receptors and Ephrins: Therapeutic Opportunities. Annual review of pharmacology and toxicology 55:465-487. DOI: 10.1146/annurev-pharmtox-011112-140226.

Byggmästar J, Granberg F, Nordlund K. 2018. Effects of the short-range repulsive potential on cascade damage in iron. Journal of Nuclear Materials 508:530-539. DOI: 10.1016/j.jnucmat.2018.06.005.

Chawla NV, Bowyer KW, Hall LO, Kegelmeyer WP. 2002. SMOTE: Synthetic Minority Over-sampling Technique. Journal of Artificial Intelligence Research 16:321-357. DOI: 10.1613/jair.953.

Chrencik JE, Brooun A, Recht MI, Nicola G, Davis LK, Abagyan R, Widmer H, Pasquale EB, Kuhn P. 2007. Three-dimensional Structure of the EphB2 Receptor in Complex with an Antagonistic Peptide Reveals a Novel Mode of Inhibition. The Journal of biological chemistry 282:3650536513. DOI: $10.1074 / j b c . M 706340200$.

drug discovery. Progress in Biophysics and Molecular Biology 116:165-173. DOI:

10.1016/j.pbiomolbio.2014.06.003. 
477 Delano WL. 2002. The PyMOL molecular graphics system.

478 Hauser AS, Attwood MM, Rask-Andersen M, Schiöth HB, Gloriam DE. 2017. Trends in GPCR drug 479 discovery: new agents, targets and indications. Nature Reviews Drug Discovery 16:829-842.

$480 \quad$ DOI: $10.1038 / \mathrm{nrd} .2017 .178$.

481 Hopkins AL, Groom CR. 2002. The druggable genome. Nature Reviews Drug Discovery 1:727-730. $482 \quad$ DOI: $10.1038 / \operatorname{nrd} 892$.

483 Işeri OD, Sahin FI, Terzi YK, Yurtcu E, Erdem SR, Sarialioglu F. 2014. beta-Adrenoreceptor antagonists 484 reduce cancer cell proliferation, invasion, and migration. Pharmaceutical Biology 52:1374-1381. $485 \quad$ DOI: $10.3109 / 13880209.2014 .892513$.

486 Ishida J, Konishi M, Ebner N, Springer J. 2016. Repurposing of approved cardiovascular drugs. Journal 487 of Translational Medicine 14. DOI: 10.1186/s12967-016-1031-5.

488

489

490

491

492

493

494

495

496

497

498

499

500

501

502

Israelachvili J, Pashley R. 1982. The hydrophobic interaction is long range, decaying exponentially with distance. Nature 300:341-342. DOI: 10.1038/300341a0.

Jabbour E, Short NJ, Ravandi F, Huang X, Xiao L, Garcia-Manero G, Plunkett W, Gandhi V, Sasaki K, Pemmaraju N, Daver NG, Borthakur G, Jain N, Konopleva M, Estrov Z, Kadia TM, Wierda WG, DiNardo CD, Brandt M, O’Brien SM, Cortes JE, Kantarjian H. 2017. A randomized phase 2 study of idarubicin and cytarabine with clofarabine or fludarabine in patients with newly diagnosed acute myeloid leukemia. Cancer 123:4430-4439. DOI: 10.1002/cncr.30883.

Kim DE, Chivian D, Baker D. 2004. Protein structure prediction and analysis using the Robetta server. Nucleic Acids Research 32:W526-531. DOI: 10.1093/nar/gkh468.

Koes DR, Dömling A, Camacho CJ. 2018. AnchorQuery: Rapid online virtual screening for small-molecule protein-protein interaction inhibitors. Protein Science : A Publication of the Protein Society 27:229-232. DOI: 10.1002/pro.3303.

Lee AC-L, Harris JL, Khanna KK, Hong J-H. 2019. A Comprehensive Review on Current Advances in Peptide Drug Development and Design. International Journal of Molecular Sciences 20. DOI: 10.3390/ijms20102383. 
503 López-Cortés A, Cabrera-Andrade A, Cruz-Segundo CM, Dorado J, Pazos A, Gonzáles-Díaz H, Paz-y-

504 Miño C, Pérez-Castillo Y, Tejera E, Munteanu CR. 2019. Prediction of druggable proteins using 505 machine learning and functional enrichment analysis: a focus on cancer-related proteins and $506 \quad$ RNA-binding proteins. bioRxiv:825513. DOI: 10.1101/825513.

507 Ma B, Nussinov R. 2014. Druggable Orthosteric and Allosteric Hot Spots to Target Protein-protein 508 Interactions. Current pharmaceutical design 20:1293-1301.

509 Malhotra U, Mukherjee S, Fountzilas C, Boland P, Miller A, Patnaik S, Attwood K, Yendamuri S, Adjei 510 A, Kannisto E, Opyrchal M, Bushunow P, Loud P, Iyer R, Khushalani N. 2020. Pralatrexate in 511 Combination with Oxaliplatin in Advanced Esophagogastric Cancer: A Phase II Trial with 512 Predictive Molecular Correlates. Molecular Cancer Therapeutics 19:304-311. DOI: 10.1158/1535-7163.MCT-19-0240.

514 515 516 517 518 519 520 521 522 523 524 525 526 527 528

Martins JM, Ramos RM, Pimenta AC, Moreira IS. 2014. Solvent-accessible surface area: How well can be applied to hot-spot detection? Proteins 82:479-490. DOI: 10.1002/prot.24413.

Martoni A, Pacciarini MA, Piana E, Pannuti F. 1986. A pilot study of oral idarubicin in metastatic melanoma. Chemioterapia: International Journal of the Mediterranean Society of Chemotherapy 5:414-415.

Modell AE, Blosser SL, Arora PS. 2016. Systematic Targeting of Protein-Protein Interactions. Trends in pharmacological sciences 37:702-713. DOI: 10.1016/j.tips.2016.05.008.

More A. 2016. Survey of resampling techniques for improving classification performance in unbalanced datasets. arXiv:1608.06048 [cs, stat].

Moreira IS, Koukos PI, Melo R, Almeida JG, Preto AJ, Schaarschmidt J, Trellet M, Gümüş ZH, Costa J, Bonvin AMJJ. 2017. SpotOn: High Accuracy Identification of Protein-Protein Interface HotSpots. Scientific Reports 7:8007. DOI: 10.1038/s41598-017-08321-2.

Noberini R, Lamberto I, Pasquale EB. 2012. Targeting Eph Receptors with Peptides and Small Molecules: Progress and Challenges. Seminars in cell \& developmental biology 23:51-57. DOI: 10.1016/j.semcdb.2011.10.023. 
529 Pantziarka P, Bouche G, Meheus L, Sukhatme V, Sukhatme VP. 2014. Repurposing drugs in oncology 530 (ReDO)-cimetidine as an anti-cancer agent. Ecancermedicalscience 8:485. DOI:

$531 \quad$ 10.3332/ecancer.2014.485.

532 Pantziarka P, Bouche G, Sukhatme V, Meheus L, Rooman I, Sukhatme VP. 2016. Repurposing Drugs in 533 Oncology (ReDO) - Propranolol as an anti-cancer agent. ecancermedicalscience 10. DOI:

$534 \quad 10.3332$ /ecancer.2016.680.

535 Pasquale EB. 2010. Eph receptors and ephrins in cancer: bidirectional signaling and beyond. Nature 536 reviews. Cancer 10:165-180. DOI: 10.1038/nrc2806.

537 Scott DE, Bayly AR, Abell C, Skidmore J. 2016. Small molecules, big targets: drug discovery faces the 538 protein-protein interaction challenge. Nature Reviews Drug Discovery 15:533-550. DOI:

$539 \quad 10.1038 /$ nrd.2016.29.

540 Serova M, Bieche I, Sablin M-P, Pronk GJ, Vidaud M, Cvitkovic E, Faivre S, Raymond E. 2011. Single 541 agent and combination studies of pralatrexate and molecular correlates of sensitivity. British $542 \quad$ Journal of Cancer 104:272-280. DOI: 10.1038/sj.bjc.6606063.

543 Tuncbag N, Keskin O, Gursoy A. 2010. HotPoint: hot spot prediction server for protein interfaces. $544 \quad$ Nucleic Acids Research 38:W402-W406. DOI: 10.1093/nar/gkq323.

545 de Vries SJ, Bonvin AMJJ. 2011. CPORT: a consensus interface predictor and its performance in 546 prediction-driven docking with HADDOCK. PloS One 6:e17695. DOI:

$547 \quad$ 10.1371/journal.pone.0017695.

548 Wang F, Liu H, Wang F, Xu R, Wang P, Tang F, Zhang X, Zhu Z, Lv H, Han T. 2018. Propranolol 549 suppresses the proliferation and induces the apoptosis of liver cancer cells. Molecular Medicine $550 \quad$ Reports 17:5213-5221. DOI: $10.3892 / \mathrm{mmr} .2018 .8476$.

551 Whalen S, Pandey G. 2013. A Comparative Analysis of Ensemble Classifiers: Case Studies in Genomics. 552 In: 2013 IEEE 13th International Conference on Data Mining. 807-816. DOI:

$553 \quad$ 10.1109/ICDM.2013.21. 
554 Wishart DS, Knox C, Guo AC, Cheng D, Shrivastava S, Tzur D, Gautam B, Hassanali M. 2008.

555 DrugBank: a knowledgebase for drugs, drug actions and drug targets. Nucleic Acids Research 556 36:D901-D906. DOI: 10.1093/nar/gkm958.

557 Wolber G, Langer T. 2005. LigandScout: 3-D pharmacophores derived from protein-bound ligands and 558 their use as virtual screening filters. Journal of Chemical Information and Modeling 45:160-169.

$559 \quad$ DOI: $10.1021 /$ ci049885e.

560 Xiao N, Cao D-S, Zhu M-F, Xu Q-S. 2015. protr/ProtrWeb: R package and web server for generating 561 various numerical representation schemes of protein sequences. Bioinformatics 31:1857-1859. $562 \quad$ DOI: 10.1093/bioinformatics/btv042.

563 Yu L, Zhao J, Gao L. 2018. Predicting Potential Drugs for Breast Cancer based on miRNA and Tissue 564 Specificity. International Journal of Biological Sciences 14:971-982. DOI: 10.7150/ijbs.23350.

565 Zhu X, Mitchell JC. 2011. KFC2: a knowledge-based hot spot prediction method based on interface

566 solvation, atomic density, and plasticity features. Proteins 79:2671-2683. DOI:

567 10.1002/prot.23094.

568

569

570 $\underline{\text { AUTHOR CONTRIBUTIONS }}$

571 Rohit Nandakumar conceived and performed the experiments as stated in this study, analyzed the data, 572 and co-authored the corresponding paper.

573 Dr. Valentin Dinu provided revisions and co-authored to this paper.

\section{ACKNOWLEDGEMENTS}

575 I would like to thank the researchers who conducted the SpotOn study, especially Ms. Irina Moreira, for 576 providing the code and existing dataset that this study is built on top of.

577 I would also like to thank Dr. Michael McKelvy of Basha High School for his extensive feedback on my 578 poster and project. 
579 In addition, I would like to thank Mr. Thomas Lemker for his assistance in using the LigandScout 580 software.

\section{SUPPLEMENTARY INFO}

582 All data used in this study is provided as the supplementary materials. 


\section{Table 1 (on next page)}

Average test metrics of algorithms tested on pre-processing pipelines 
1 Table 1: Average test metrics of algorithms tested on pre-processing pipelines

\begin{tabular}{|c|c|c|c|c|c|c|c|c|c|}
\hline Test & AUROC & Accuracy & $\begin{array}{l}\text { Precisio } \\
\mathrm{n}\end{array}$ & $\begin{array}{l}\text { Recall/ } \\
\text { Sensitiv } \\
\text { ity }\end{array}$ & F1 & $\begin{array}{l}\text { Precision } \\
\text {-Recall }\end{array}$ & $\mathrm{MCC}$ & Kappa & Specificity \\
\hline $\begin{array}{l}\text { ONLY } \\
\text { SMOTE }\end{array}$ & 0.745 & 0.772 & 0.516 & 0.694 & 0.584 & 0.430 & 0.451 & 0.435 & 0.796 \\
\hline RAW & 0.722 & 0.777 & 0.536 & 0.618 & 0.563 & 0.416 & 0.427 & 0.418 & 0.825 \\
\hline $\begin{array}{l}\text { NO } \\
\text { SMOTE } \\
\text {, PCA }\end{array}$ & 0.702 & 0.785 & 0.535 & 0.549 & 0.539 & 0.403 & 0.402 & 0.400 & 0.856 \\
\hline $\begin{array}{l}\text { SMOTE } \\
\text {, PCA }\end{array}$ & 0.774 & 0.798 & 0.551 & 0.729 & 0.625 & 0.467 & 0.502 & 0.491 & 0.819 \\
\hline
\end{tabular}

2 
Table 2 (on next page)

Best Individual Algorithms in SMOTE and PCA pipeline 
1 Table 2: Best Individual Algorithms in SMOTE and PCA pipeline

\begin{tabular}{|l|l|l|l|l|l|l|l|l|l|}
\hline & AUROC & Accuracy & Precision & Recall/ & F1 & Precision- & MCC & Kappa & Specificity \\
& & & & Sensitivity & & Recall & & & \\
\hline LR & $\mathbf{0 . 8 4 2}$ & 0.846 & 0.625 & 0.833 & 0.714 & 0.559 & 0.624 & 0.612 & 0.850 \\
\hline RF & $\mathbf{0 . 7 5 6}$ & 0.827 & 0.625 & 0.625 & 0.625 & 0.477 & 0.513 & 0.513 & 0.888 \\
\hline GBC & $\mathbf{0 . 7 4 8}$ & 0.769 & 0.500 & 0.708 & 0.586 & 0.422 & 0.445 & 0.433 & 0.788 \\
\hline KNN & $\mathbf{0 . 7 0 6}$ & 0.750 & 0.469 & 0.625 & 0.536 & 0.380 & 0.377 & 0.369 & 0.788 \\
\hline MLP & $\mathbf{0 . 8 2 9}$ & 0.827 & 0.588 & 0.833 & 0.690 & 0.529 & 0.591 & 0.575 & 0.825 \\
\hline Gaussian & $\mathbf{0 . 7 5 6}$ & 0.827 & 0.625 & 0.625 & 0.625 & 0.477 & 0.513 & 0.513 & 0.888 \\
\hline
\end{tabular}

2 
Table 3 (on next page)

Comparison of our study vs SpotOn 
1 Table 3: Comparison of our study vs SpotOn

\begin{tabular}{|l|l|l|}
\hline Test & LG from & SpotOn's \\
& PCA and & ScaledUp* \\
\hline Accuracy & $\mathbf{0 . 8 6 5}$ & 0.79 \\
\hline F1 & $\mathbf{0 . 7 3 1}$ & 0.52 \\
\hline AUROC & $\mathbf{0 . 8 4 0}$ & 0.83 \\
\hline MCC & $\mathbf{0 . 6 4 5}$ & 0.38 \\
\hline Sensitivity & $\mathbf{0 . 7 9 2}$ & 0.48 \\
\hline Specificity & $\mathbf{0 . 8 8 8}$ & 0.88 \\
\hline
\end{tabular}

2 *This data was adapted from the SpotOn study

3 
Table 4 (on next page)

Different ensemble classifiers (stacking and voting) were tested 
1 Table 4: Different ensemble classifiers (stacking and voting) were tested

2

\begin{tabular}{|c|c|c|c|c|c|c|c|c|c|}
\hline & AUROC & Accuracy & Precision & $\begin{array}{l}\text { Recall/ } \\
\text { Sensitivity }\end{array}$ & $\mathrm{F} 1$ & $\begin{array}{l}\text { Precision } \\
\text {-Recall }\end{array}$ & $\mathrm{MCC}$ & Kappa & Specificity \\
\hline LR w/ Logistic & & & & & & & & & \\
\hline Regression & 0.819 & 0.789 & 0.525 & 0.875 & 0.656 & 0.488 & 0.552 & 0.517 & 0.763 \\
\hline RF w/ Logistic & & & & & & & & & \\
\hline Regression & 0.729 & 0.740 & 0.460 & 0.708 & 0.557 & 0.393 & 0.403 & 0.385 & 0.750 \\
\hline GBC w/ Logistic & & & & & & & & & \\
\hline Regression & 0.773 & 0.808 & 0.567 & 0.708 & 0.630 & 0.469 & 0.508 & 0.502 & 0.838 \\
\hline KNN w/ Logistic & & & & & & & & & \\
\hline Regression & 0.656 & 0.673 & 0.375 & 0.625 & 0.469 & 0.321 & 0.271 & 0.253 & 0.688 \\
\hline MLP w/ Logistic & & & & & & & & & \\
\hline Regression & 0.798 & 0.779 & 0.513 & 0.833 & 0.635 & 0.466 & 0.519 & 0.489 & 0.763 \\
\hline Gaussian w/ & & & & & & & & & \\
\hline Logistic & & & & & & & & & \\
\hline Regression & 0.581 & 0.490 & 0.277 & 0.750 & 0.405 & 0.265 & 0.141 & 0.102 & 0.413 \\
\hline All (Stacking) w/ & & & & & & & & & \\
\hline Logistic & & 0.827 & & & & & & & \\
\hline Regression & 0.785 & & 0.607 & 0.708 & 0.654 & 0.497 & 0.542 & 0.539 & 0.863 \\
\hline Voting Classifier & 0.710 & 0.712 & 0.425 & 0.708 & 0.531 & 0.368 & 0.365 & 0.341 & 0.713 \\
\hline
\end{tabular}




\section{Table 5 (on next page)}

Comparison of our study to other studies 
1 Table 5: Comparison of our study to other studies

\begin{tabular}{|l|l|l|l|l|l|l|l|}
\hline & Our & SpotOn* & SBHD2* & Robetta* & KFC2- & KFC2- & CPORT* \\
& & & & & A* & \\
\hline AUROC & $\mathbf{0 . 8 4 2}$ & 0.91 & 0.69 & 0.62 & 0.66 & 0.67 & 0.54 \\
\hline Sensitivity & $\mathbf{0 . 8 3 3}$ & 0.98 & 0.7 & 0.29 & 0.53 & 0.28 & 0.54 \\
\hline Specificity & $\mathbf{0 . 8 5 0}$ & 0.84 & 0.71 & 0.88 & 0.81 & 0.96 & 0.47 \\
\hline F1-score & $\mathbf{0 . 7 1 4}$ & 0.96 & 0.62 & 0.39 & 0.56 & 0.42 & 0.42 \\
\hline
\end{tabular}

2 * Columns 2 through 7 are adapted from the SpotOn study to perform the side-by-side comparison among the algorithms 3 
Table 6(on next page)

Pharmacophore fit rankings from drug screening: Details for top ranked drugs 
1 Table 6: Pharmacophore fit rankings from drug screening: Details for top ranked drugs

\begin{tabular}{|l|l|l|l|}
\hline Generic Name & $\begin{array}{l}\text { Pharmacophore } \\
\text { Fit Score }\end{array}$ & $\begin{array}{l}\text { Database } \\
\text { ID }\end{array}$ & $\begin{array}{l}\text { Molecular } \\
\text { Weight }\end{array}$ \\
\hline Pralatrexate & 47.41 & DB06813 & 477.47 \\
\hline Chlortetracycline & 46.02 & DB09093 & 478.88 \\
\hline Nadolol & 45.97 & DB01203 & 309.4 \\
\hline Imipenem & 45.51 & DB01598 & 299.35 \\
\hline Idarubicin & 45.46 & DB01177 & 497.49 \\
\hline Valganciclovir & 44.76 & DB01610 & 354.36 \\
\hline Conivaptan & 43.92 & DB00872 & 498.57 \\
\hline Cimetidine & 43.86 & DB00501 & 252.34 \\
\hline Barnidipine & 43.53 & DB09227 & 491.54 \\
\hline
\end{tabular}

2 


\section{Figure 1}

Feature importances of the top logistic regression classifier

Sensitivity analysis of the logistic regression from the SMOTE-only pipeline were performed.

Features near the top of the graph have higher feature importances. 


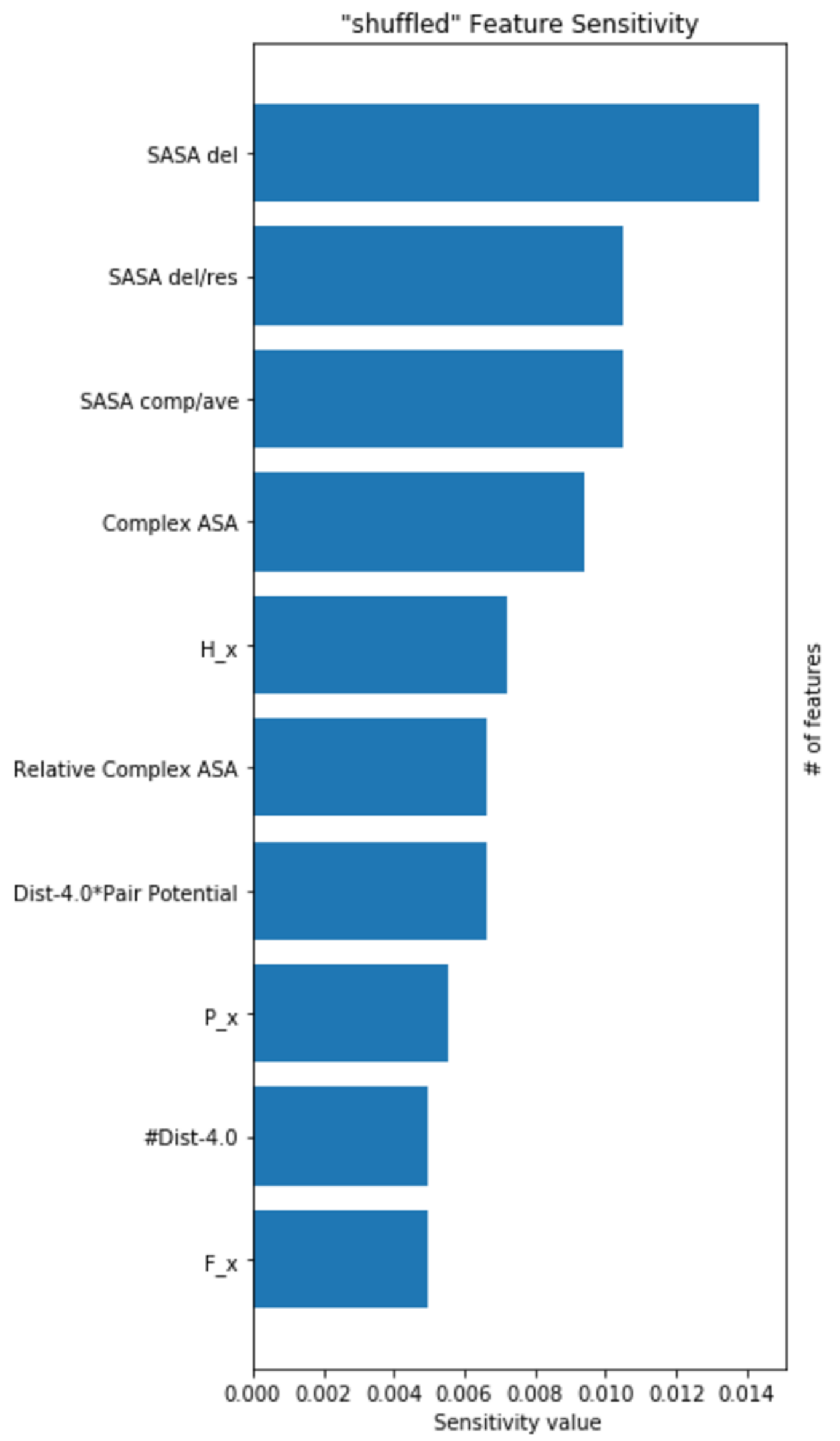


Figure 2

The EphB2-ephrinB2 complex with highlighted residues using PyMol

Residues 1122-1126 are highlighted as shown in green as surface markers. The rest of the complex is in pink.

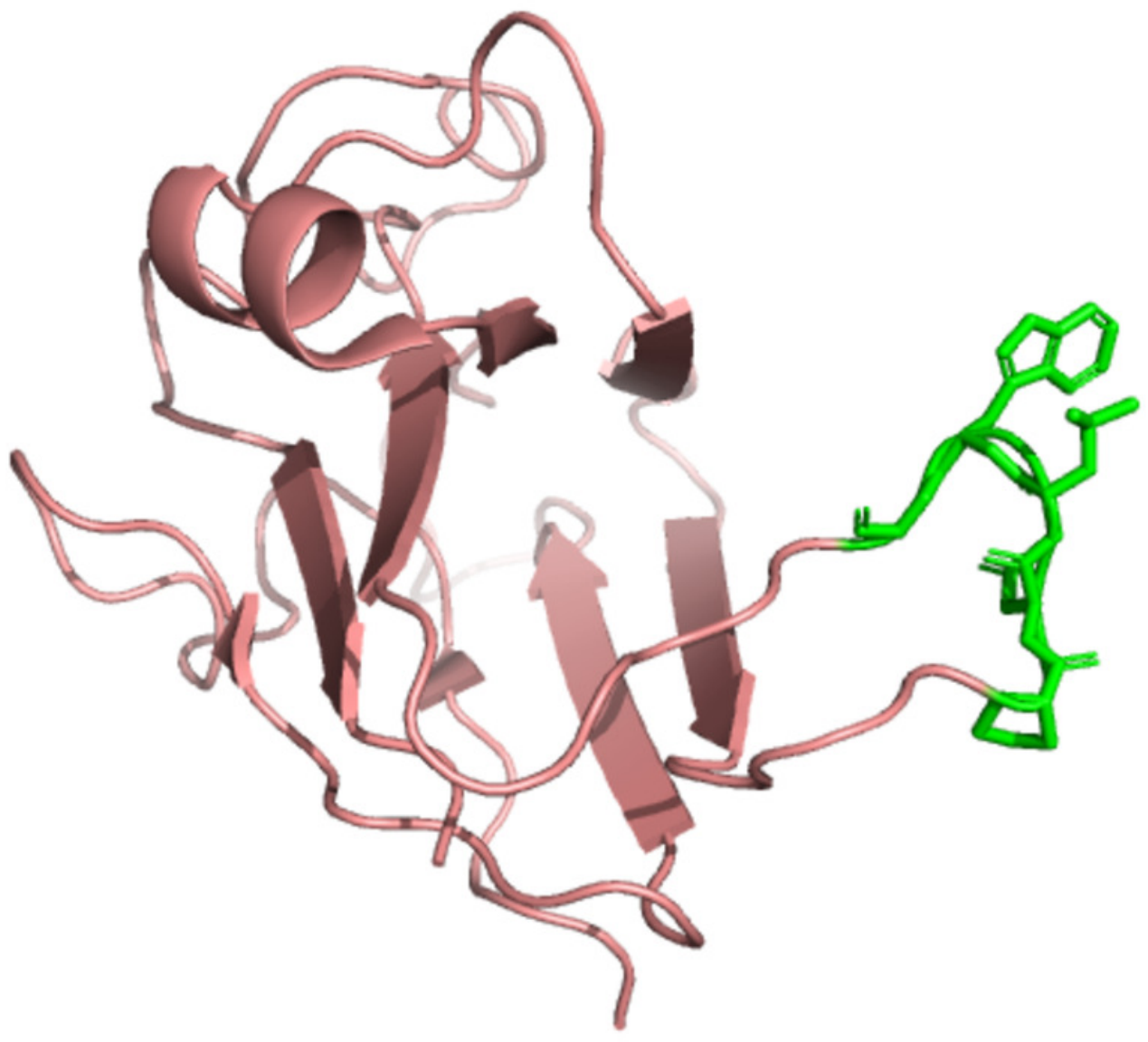


Figure 3

Apo-Site Grid for residues 1122-1126

Apo site pharmacophore of residues $1122-1126$. The gray parts of the grid indicate the levels of buriedness and surface area.

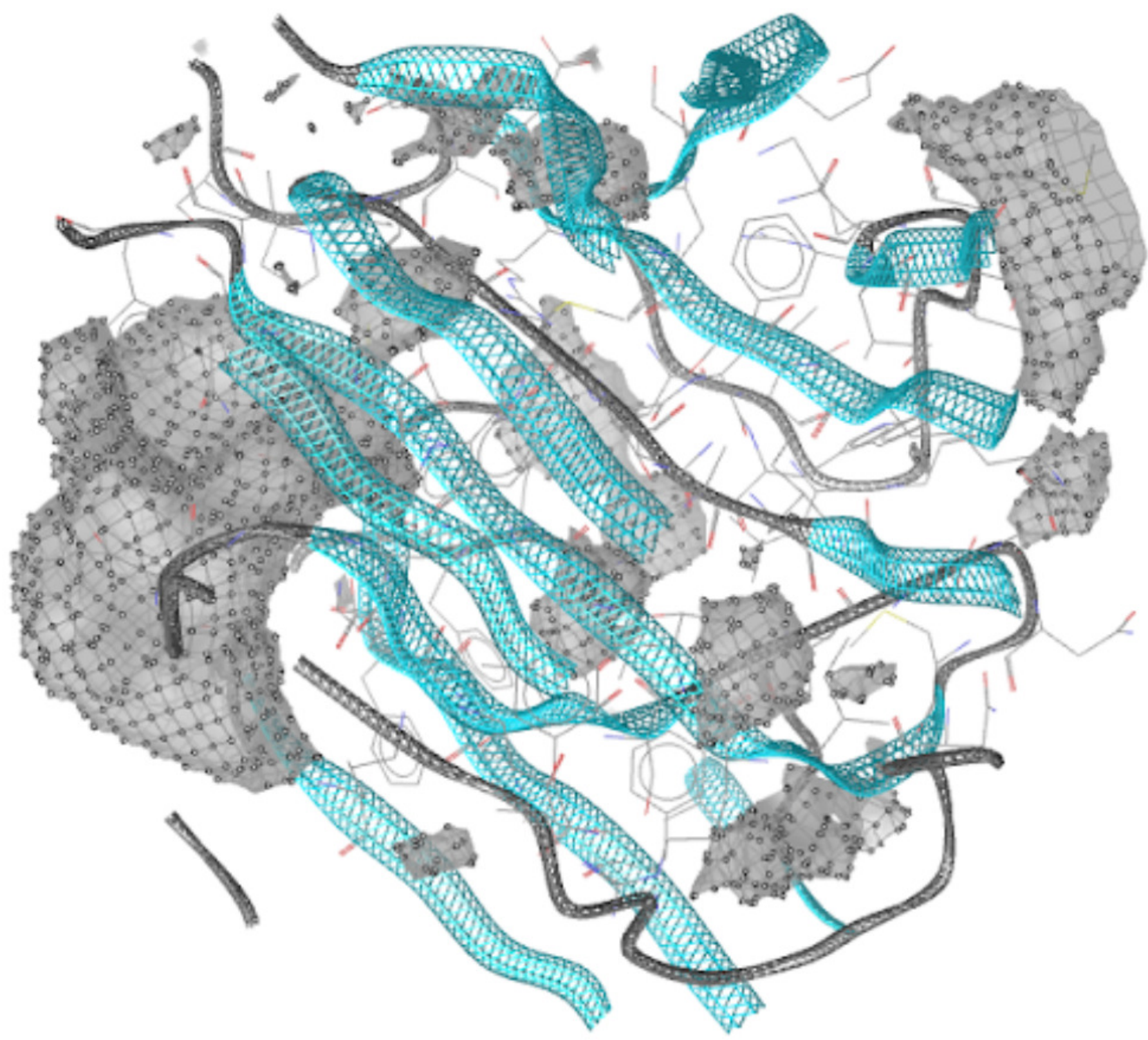


Figure 4

Pharmacophore model of residues 1122-1126

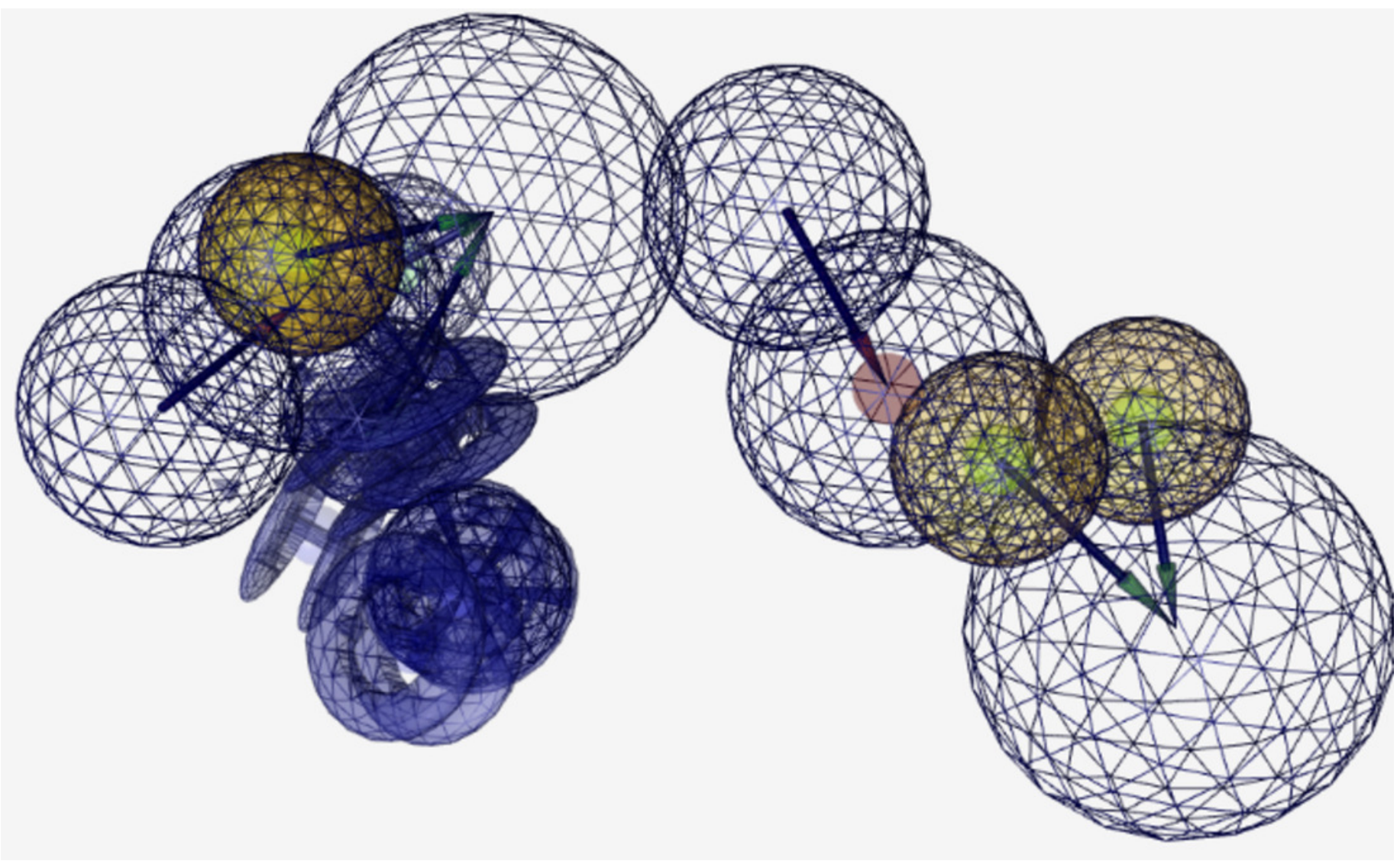


Figure 5

Structure and relative structure of cimetidine in relation to the developed pharmacophore
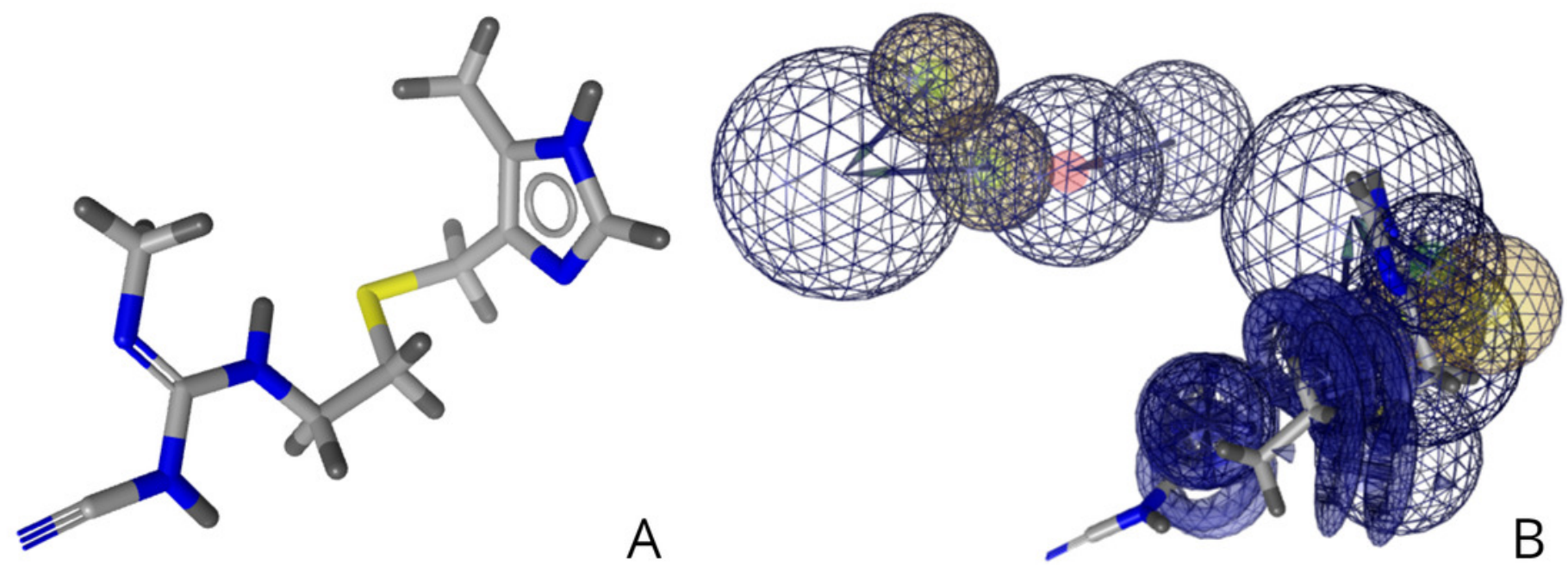
Figure 6

Structure and relative structure of idarubicin in relation to the developed pharmacophore

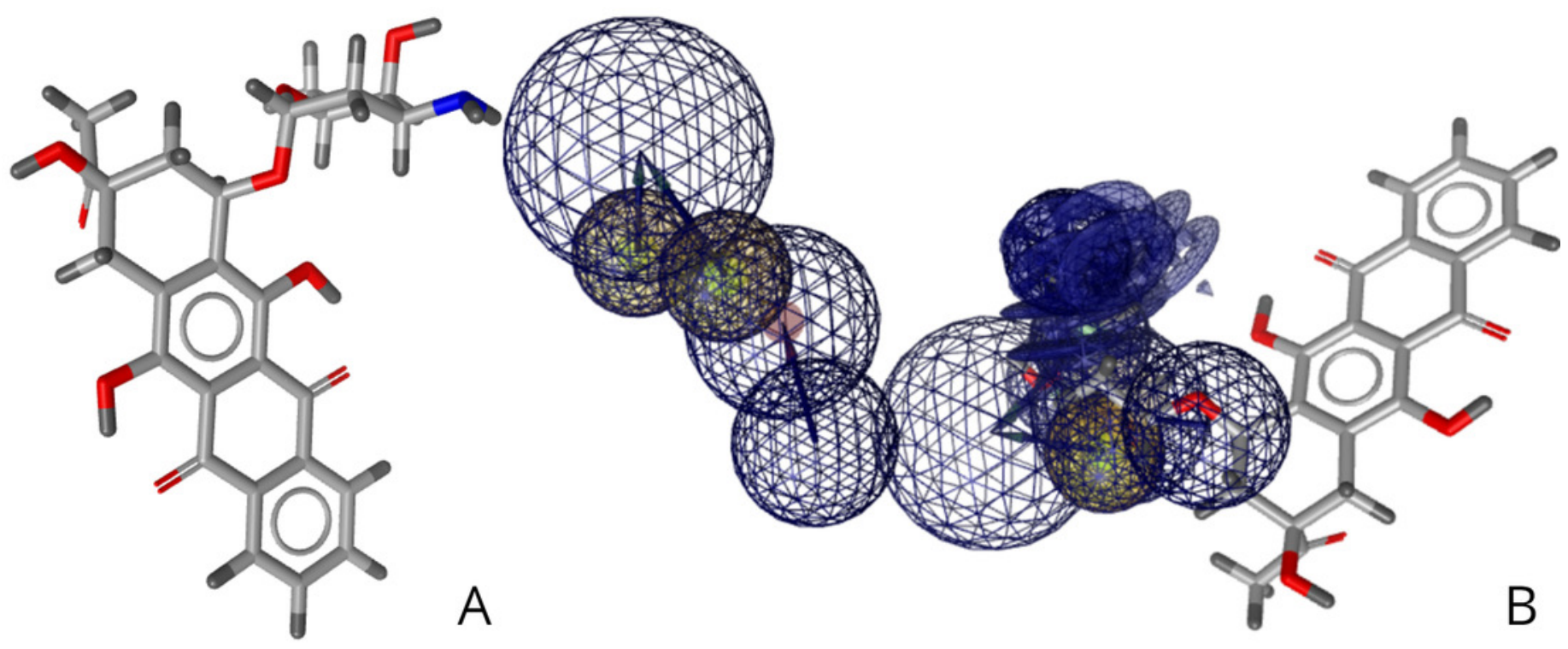


Figure 7

Structure and relative structure of pralatrexate in relation to the developed pharmacophore
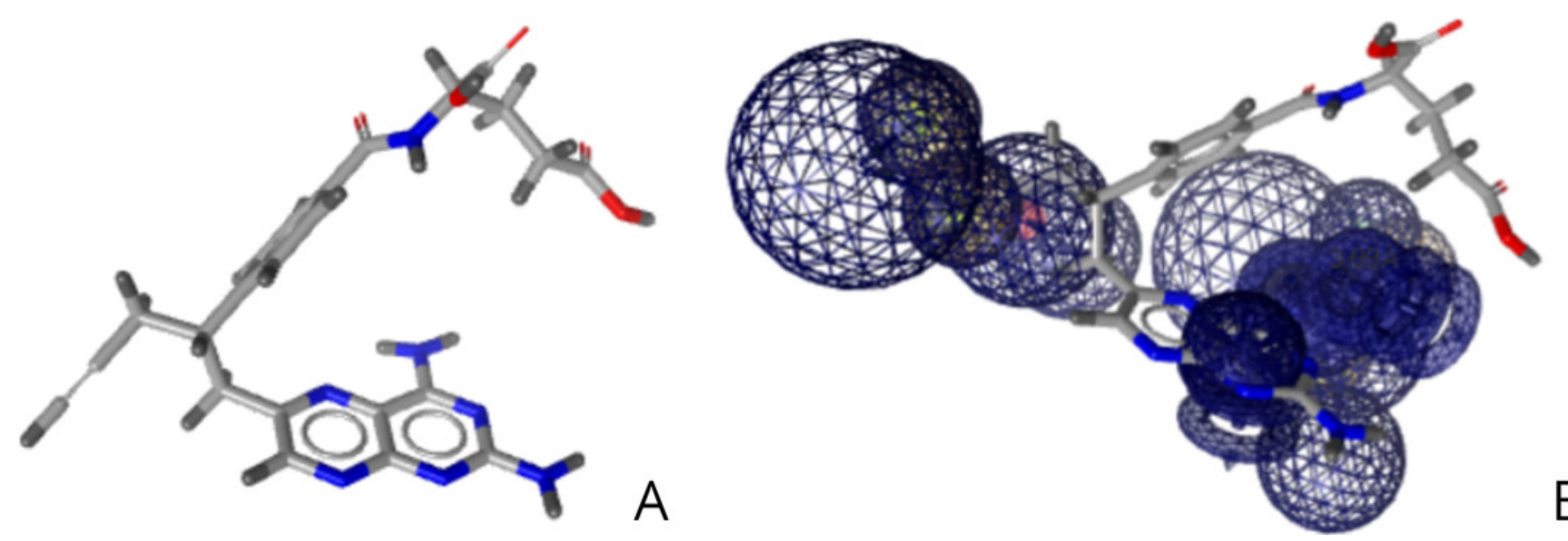
Figure 8

Structure and relative structure of nadolol in relation to the developed pharmacophore
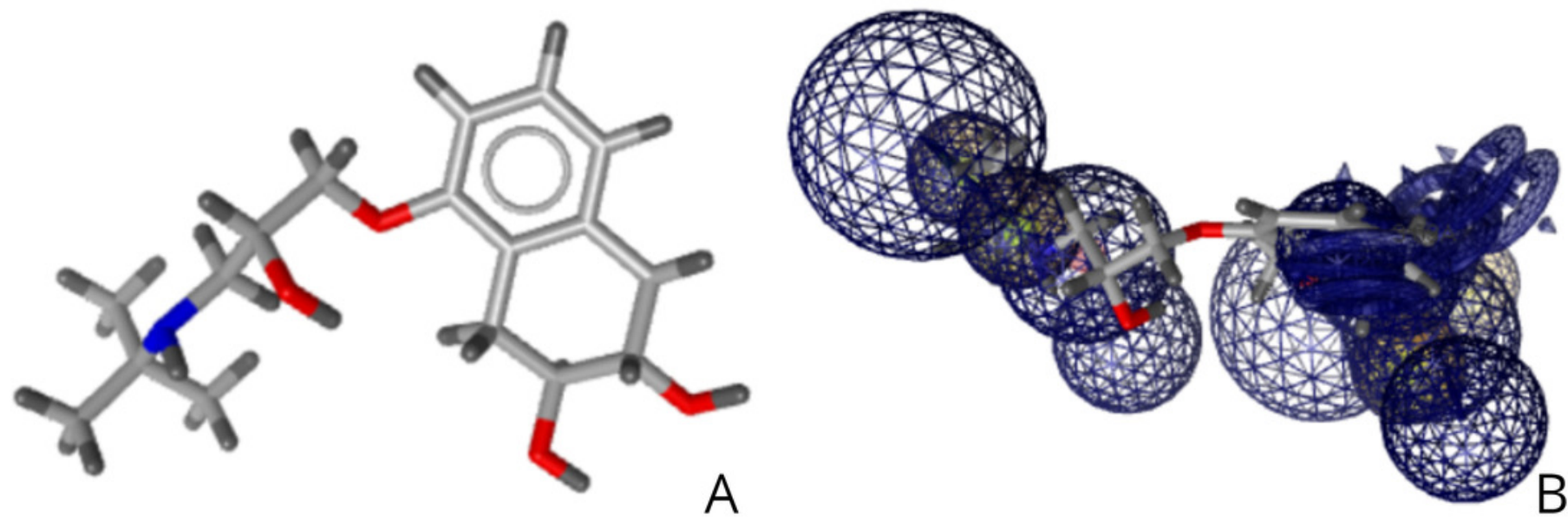